\title{
An extension of Furuta's log majorization inequality
}

\author{
Yanbo Ren ${ }^{\mathrm{a}}$, Jian $\mathrm{Shi}^{\mathrm{b}, *}$ \\ a School of Mathematics and Statistics, Henan University of Science and Technology, Luoyang, 471023, P. R. China. \\ ${ }^{b}$ College of Mathematics and Information Science, Hebei University, Baoding, 071002, P. R. China.
}

Communicated by Y. J. Cho

\begin{abstract}
In this paper, we shall prove a log majorization inequality, which extends Furuta's result. (C)2017 All rights reserved.
\end{abstract}

Keywords: Log majorization, Koizumi-Watanable inequality. 2010 MSC: 47A63.

\section{Introduction}

A capital letter, such as $T$, stands for a bounded linear operator on a Hilbert space. The notation $T \geqslant 0$ means that $T$ is positive semidefinite and $T>0$ means that $T$ is positive definite.

Definition 1.1 ([1]). Log majorization for two positive semidefinite $n \times n$ matrices $A$ and $B$, denoted by $A \succ{ }_{(\log )} B$, if $\prod_{i=1}^{k} \lambda_{i}(A) \geqslant \prod_{i=1}^{k} \lambda_{i}(B)$ for $k=1,2, \cdots, n-1$ and $\prod_{i=1}^{n} \lambda_{i}(A)=\prod_{i=1}^{n} \lambda_{i}(B)$, where $\lambda_{1}(A) \geqslant \lambda_{2}(A) \geqslant \cdots \geqslant \lambda_{n}(A)$ and $\lambda_{1}(B) \geqslant \lambda_{2}(B) \geqslant \cdots \geqslant \lambda_{n}(B)$ are the eigenvalues of $A$ and $B$, respectively.

Definition 1.2 ([6]). For $A, B>0$, Kubo-Ando mean of $A$ and $B$ for $\alpha$ power is defined by

$$
A^{\frac{1}{2}}\left(A^{-\frac{1}{2}} B A^{-\frac{1}{2}}\right)^{\alpha} A^{\frac{1}{2}}
$$

which is denoted by $A \sharp \alpha B$, where $\alpha \in[0,1]$;

If $A, B \geqslant 0, A \sharp_{\alpha} B$ is defined by $\lim _{\varepsilon \rightarrow 0^{+}}(A+\varepsilon I) \sharp_{\alpha}(B+\varepsilon I)$;

If $A>0, B \geqslant 0$ with $\alpha \in \mathbb{R}, A^{\frac{1}{2}}\left(A^{-\frac{1}{2}} B A^{-\frac{1}{2}}\right)^{\alpha} A^{\frac{1}{2}}$ is denoted by $A \natural_{\alpha} B$.

In 1994, Ando and Hiai proved the first log majorization inequality as follows, which is also called Ando-Hiai inequality.

Theorem 1.3 ([1, Ando-Hiai inequality $])$. If $A, B \geqslant 0,0 \leqslant \alpha \leqslant 1$, then $\left(A \sharp_{\alpha} B\right)^{r} \succ_{(\log )} A^{r} \sharp_{\alpha} B^{r}$ holds for $r \geqslant 1$.

\footnotetext{
*Corresponding author

Email addresses: ryb7945@sina.com (Yanbo Ren), mathematic@126.com (Jian Shi)
} 
In 1995, Furuta generalized Ando-Hiai inequality and obtained the following theorem.

Theorem 1.4 ([2]). If $A, B \geqslant 0,0 \leqslant \alpha \leqslant 1$, then $\left(A \sharp_{\alpha} B\right)^{h} \succ_{(\log )} A^{r} \sharp_{\frac{h}{s}} B^{s}$ holds for $r, s \geqslant 1$ with $h=$ $\left(\alpha s^{-1}+(1-\alpha) r^{-1}\right)^{-1}$.

Subsequently, various log majorization inequalities were shown, such as [3,4,7]. One of the most wonderful result is proved by Furuta ([3]) in 2009 as follows.

Theorem 1.5 ([3]). For $A>0, B \geqslant 0, t \in[0,1]$ and $r \geqslant t, p_{1}, p_{2}, \cdots, p_{2 n} \geqslant 1$, then

$$
\begin{aligned}
& \left(A \sharp \frac{1}{p_{1}} B\right)^{h} \succ(\log ) \\
& A^{1-t+r_{\sharp}} \sharp_{\beta}\left\{A^{1-t} \natural_{p_{2 n}}\left\{A \natural_{p_{2 n-1}}\left\{A^{1-t} \natural_{p_{2 n-2}}\left\{A \natural_{p_{2 n-3}} \cdots\left[A \natural_{p_{3}}\left(A^{1-t} \natural_{p_{2}} B\right)\right] \cdots\right\}\right\}\right\}\right\}
\end{aligned}
$$

holds, where $h=\frac{p_{1} p_{2} \cdots p_{2 n}(1-t+r)}{\phi}, \beta=\frac{h}{p_{1} p_{2} \cdots p_{2 n}}$, with $\phi=\left[\cdots\left\{\left[\left(p_{1}-t\right) p_{2}+t\right] p_{3}-t\right\} p_{4}+t \cdots-t\right] p_{2 n}+r$.

In this paper, we shall show an extension of Theorem 1.5. In order to prove the main results, we shall list a useful theorem first.

Theorem 1.6 ([5, Koizumi-Watanabe inequality]). For $A>0, B \geqslant 0, t_{2 k-1} \in[0,1]$ and $t_{2 k-1} \leqslant t_{2 k}$ for $k=1,2, \cdots, n, p_{1}, p_{2}, \cdots, p_{2 n} \geqslant 1$, then

$$
\left\{A^{\frac{t_{2 n}}{2}}\left[A^{-\frac{t_{2 n-1}}{2}} \cdots\left[A^{\frac{t_{2}}{2}}\left(A^{-\frac{t_{1}}{2}} B^{p_{1}} A^{-\frac{t_{1}}{2}}\right)^{p_{2}} A^{\frac{t_{2}}{2}}\right]^{p_{3}} \cdots A^{-\frac{t_{2 n-1}}{2}}\right]^{p_{2 n}} A^{\frac{t_{2 n}}{2}}\right\}^{\frac{\alpha(2 n)}{\phi(2 n)}} \leqslant A^{\alpha(2 n)}
$$

holds for $\alpha(2 n)=1-t_{1}+t_{2} \cdots-t_{2 n-1}+t_{2 n}, \phi(2 n)=\left[\cdots\left\{\left[\left(p_{1}-t_{1}\right) p_{2}+t_{2}\right] p_{3}-t_{3}\right\} p_{4}+t_{4} \cdots-t_{2 n-1}\right] p_{2 n}+$ $t_{2 n}$.

\section{Main results}

By using the method in [1] and [3], we can show the main result.

Theorem 2.1. For $\mathrm{A}>0, \mathrm{~B} \geqslant 0$, the following $\log$ majorization inequality

$$
\begin{aligned}
& \left(A \sharp_{\alpha} B\right)^{h} \succ(\log ) \\
& A^{\alpha(2 n)} \sharp_{\beta}\left\{A^{\alpha(2 n-1)} \bigsqcup_{p_{2 n}}\left\{A^{\alpha(2 n-2)} \bigsqcup_{p_{2 n-1}}\left\{A^{\alpha(2 n-3)} \bigsqcup_{p_{2 n-2}} \cdots\left[A^{\alpha(2)} \bigsqcup_{p_{3}}\left(A^{\alpha(1)} \bigsqcup_{p_{2}} B\right)\right] \cdots\right\}\right\}\right\}
\end{aligned}
$$

holds for $\alpha=\frac{1}{p_{1}}, \beta=\frac{\alpha(2 n)}{\phi(2 n)}, h=\frac{p_{1} p_{2} \cdots p_{2 n} \alpha(2 n)}{\phi(2 n)}$ with $\alpha(k)=1-t_{1}+t_{2} \cdots+(-1)^{k} t_{k}, \phi(2 n)=\left[\cdots\left\{\left[\left(p_{1}-\right.\right.\right.\right.$ $\left.\left.\left.\left.t_{1}\right) p_{2}+t_{2}\right] p_{3}-t_{3}\right\} p_{4}+t_{4} \cdots-t_{2 n-1}\right] p_{2 n}+t_{2 n}$, where $t_{2 k-1} \in[0,1]$ and $t_{2 k-1} \leqslant t_{2 k}$ for $k=1,2, \cdots, n$; $p_{1}, p_{2}, \cdots, p_{2 n} \geqslant 1$.

Proof. In order to prove (2.1), we only need to prove that $I \geqslant A \sharp_{\alpha} B$ ensures that

$$
I \geqslant A^{\alpha(2 n)} \sharp_{\beta}\left\{A^{\alpha(2 n-1)} \bigsqcup_{p_{2 n}}\left\{A^{\alpha(2 n-2)} \natural_{p_{2 n-1}}\left\{A^{\alpha(2 n-3)} \bigsqcup_{p_{2 n-2}} \cdots\left[A^{\alpha(2)} \natural_{p_{3}}\left(A^{\alpha(1)} \bigsqcup_{p_{2}} B\right)\right] \cdots\right\}\right\}\right\} .
$$

By the definition of $\sharp$ and $\downarrow, I \geqslant A \sharp_{\alpha} B$ is equivalent to $A^{-1} \geqslant\left(A^{-\frac{1}{2}} B A^{-\frac{1}{2}}\right)^{\alpha}$ and (2.2) is equivalent to

$$
\begin{aligned}
A^{-\alpha(2 n)} \geqslant & \left\{A ^ { - \frac { t _ { 2 n } } { 2 } } \left[A ^ { \frac { t _ { 2 n - 1 } } { 2 } } \left(A^{-\frac{t_{2 n-2}}{2}} \cdots\right.\right.\right. \\
& \left.\left.\left.\left(A^{\frac{t_{3}}{2}}\left(A^{-\frac{t_{2}}{2}}\left(A^{-\frac{1-t_{1}}{2}} B A^{-\frac{1-t_{1}}{2}}\right)^{p_{2}} A^{-\frac{t_{2}}{2}}\right)^{p_{3}} A^{\frac{t_{3}}{2}}\right)^{p_{4}} \cdots A^{-\frac{t_{2 n-2}}{2}}\right)^{p_{2 n-1}} A^{\frac{t_{2 n-1}}{2}}\right]^{p_{2 n} n} A^{-\frac{t_{2 n}}{2}}\right\}^{\beta} .
\end{aligned}
$$

Let $A_{1}=A^{-1}$ and $B_{1}=\left(A^{-\frac{1}{2}} B A^{-\frac{1}{2}}\right)^{\alpha}$. Apply $A_{1} \geqslant B_{1}$ to Koizumi-Watanabe inequality, then

$$
A_{1}^{\alpha(2 n)} \geqslant\left\{A_{1}^{\frac{t_{2 n}}{2}}\left[A_{1}^{-\frac{t_{2 n-1}}{2}} \cdots\left[A_{1}^{\frac{t_{2}}{2}}\left(A_{1}^{-\frac{t_{1}}{2}} B_{1}^{p_{1}} A_{1}^{-\frac{t_{1}}{2}}\right)^{p_{2}} A_{1}^{\frac{t_{2}}{2}}\right]^{p_{3}} \cdots A_{1}^{-\frac{t_{2 n-1}}{2}}\right]^{p_{2 n}} A_{1}^{\frac{t_{2 n}}{2}}\right\}^{\beta} .
$$

Replacing $A_{1}$ by $A^{-1}$ and $B_{1}$ by $\left(A^{-\frac{1}{2}} B A^{-\frac{1}{2}}\right)^{\alpha}$ above, respectively, then we can obtain (2.3). 
Remark 2.2. If $t_{1}=t_{2}=\cdots=t_{2 n-1}=t$, then Theorem 2.1 is just Theorem 1.5 , which is the main result of [3].

From the proof of Theorem 2.1, we can notice that (2.1) is derived from Koizumi-Watanabe inequality. The next theorem shows that (2.1) and Koizumi-Watanabe inequality are equivalent.

Theorem 2.3. For $\mathrm{A}>0$ and $\mathrm{B} \geqslant 0$, Theorem 2.1 and Koizumi-Watanabe inequality are equivalent each other under the conditions of Theorem 2.1.

Proof. We only need to prove that Koizumi-Watanabe inequality can be derived from Theorem 2.1.

For $A>0$ and $B \geqslant 0,(2.1)$ means that $I \geqslant A \sharp_{\alpha} B$ ensures (2.2). It follows that $A^{-1} \geqslant\left(A^{-\frac{1}{2}} B A^{-\frac{1}{2}}\right)^{\alpha}$ ensures (2.3).

Replacing $A$ by $A_{1}^{-1}$ and $B$ by $A_{1}^{-\frac{1}{2}} B_{1} A_{1}^{-\frac{1}{2}}$ in (2.3), then $A_{1} \geqslant B_{1} \geqslant 0$ with $A_{1}>0$ ensure

$$
A_{1}^{\alpha(2 n)} \geqslant\left\{A_{1}^{\frac{t_{2 n}}{2}}\left[A_{1}^{-\frac{t_{2 n-1}}{2}} \cdots\left[A_{1}^{\frac{t_{2}}{2}}\left(A_{1}^{-\frac{t_{1}}{2}} B_{1}^{p_{1}} A_{1}^{-\frac{t_{1}}{2}}\right)^{p_{2}} A_{1}^{\frac{t_{2}}{2}}\right]^{p_{3}} \cdots A_{1}^{-\frac{t_{2 n}-1}{2}}\right]^{p_{2 n} n} A_{1}^{\frac{t_{2 n}}{2}}\right\}^{\beta} .
$$

The inequality above is just Koizumi-Watanabe inequality.

\section{Acknowledgment}

Yanbo Ren is supported by the National Natural Science Foundation of China (No. 11301152) and Scientific Research Foundation for Doctoral Scholars of Haust (09001772). The corresponding author Jian Shi is supported by Hebei Education Department(ZC2016009), Natural Science Foundation of Shandong Province (No. BS2015SF006).

\section{References}

[1] T. Ando, F. Hiai, Log majorization and complementary Golden-Thompson type inequalities, Second Conference of the International Linear Algebra Society (ILAS) (Lisbon, 1992), Linear Algebra Appl., 197 (1994), 113-131. 1.1, 1.3, 2

[2] T. Furuta, Extension of the Furuta inquality and Ando-Hiai log majorization, Linear Algebra Appl., 219 (1995), $139-155$. 1.4

[3] T. Furuta, Log majorization via an order preserving operator inequality, Linear Algebra Appl., 431 (2009), 132-138. 1, $1.5,2,2.2$

[4] F. Hiai, A generalization of Araki's log-majorization, Linear Algebra Appl., 501 (2016), 1-16. 1

[5] T. Koizumi, K. Watanabe, A remark on extension of order preserving operator inequality, J. Math. Inequal., 6 (2012), 119-124. 1.6

[6] F. Kubo, T. Ando, Means of positive linear operators, Math. Ann., 246 (1979/80), 205-224. 1.2

[7] R. Pereira, Weak log-majorization, Mahler measure and polynomial inequalities, Linear Algebra Appl., 421 (2007), 117121. 1 